\title{
Rapid and Sensitive Detection of Lophophora williamsii by Loop-Mediated Isothermal Amplification
}

\author{
Yohei Sasaki, ${ }^{a}$ Tsuguto Fujimoto, ${ }^{b}$ Masako Aragane, ${ }^{c}$ Ichiro Yasuda, ${ }^{d}$ and Seiji Nagumo ${ }^{*}, a$ \\ ${ }^{a}$ Department of Medicinal Plant Science, Hoshi University; 2-4-41 Ebara, Shinagawa, Tokyo 142-8501, Japan. \\ ${ }^{b}$ National Institute of Infectious Disease; 1-23-1 Toyama, Shinjuku, Tokyo 162-8640, Japan: ${ }^{c}$ Medicinal Plant Garden, \\ Tokyo Metropolitan Institute of Public Health; 21-1 Nakajima-cho, Kodaira, Tokyo 187-0033, Japan: and ${ }^{d}$ Laboratory of \\ Public Health, Faculty of Pharmacy, Chiba Institute of Science; 15-8 Shiomi-cho, Choshi, Chiba 288-0025, Japan. \\ Received December 24, 2008; accepted February 6, 2009; published online February 16, 2009
}

\begin{abstract}
We have developed a convenient method for the detection of Lophophora williamsii using loop-mediated isothermal amplification (LAMP). We designed six species-specific primers for $L$. williamsii, including two loop primers. This $L$. williamsii-specific primer set was used for LAMP of total DNA extracted from $L$. williamsii and from $L$. diffusa. Real-time monitoring of LAMP was achieved by measuring turbidity due to the formation of magnesium pyrophosphate. Amplification occurred in samples mixed with total DNA from L. williamsii, but not in those mixed with total DNA from $L$. diffusa. We could also visually observe $L$. williamsii by adding fluorescent detection reagent to the reaction tube and exposing it to UV light. This new method amplified $L$. williamsii selectively and is expected to be applicable to the detection of peyote.
\end{abstract}

Key words Lophophora williamsii; Cactaceae; peyote; visual detection; loop-mediated isothermal amplification; trnL intron

In plants, nucleotide alignment of specific DNA regions, such as nuclear ribosomal RNA or chloroplast DNA, has been used for the determination of phylogenetic relationships and species classification. ${ }^{1-3)}$ Such DNA information has also been used to authenticate herbal medicine in pharmacognosy. ${ }^{4,5}$ There have been attempts to use DNA polymorphism-based detection techniques - commonly employed in the life sciences - for the authentication of medicinal plants. Such techniques include amplification-refractory mutation system (ARMS), ${ }^{6}$ polymerase chain reaction (PCR)-restriction fragment-length polymorphism (PCR-RFLP), ${ }^{7)}$ and TaqMan assay. ${ }^{8)}$ However, not all techniques can be applied directly to plant materials because total DNA from plants contain many secondary metabolites and polysaccharides. In PCR amplification, difficulties are sometimes encountered when total DNA extracted from plant materials was used as template without preliminary preparation of the sample via purification or technical modification. ${ }^{9,10)}$ Moreover, the sequence selectivity of oligonucleotides such as PCR primers is decreased in reaction solutions that contain impurities. As many herbal medicines are derived from underground parts having high polysaccharide content, we saw the need to develop a new method to overcome this problem.

Loop-mediated isothermal amplification (LAMP) technique has been developed and used in the detection of microorganisms. ${ }^{11-13)}$ As LAMP employs 4 to 6 primers, it involves 4 to 6 reactive sites and 6 to 8 recognition regions, giving it increased sensitivity and specificity compared to typical PCR. Moreover, the isothermal reaction condition increases time efficiency compared to PCR, which requires three temperature steps. LAMP achieves detection in one to two hours. We have already observed that LAMP has good amplification ability in the detection and identification of Curcuma plants. ${ }^{14)}$ LAMP was successful even though Curcuma rhizomes have high polysaccharide content, which made extraction of total DNA difficult.

The genus Lophophora, Cactaceae, is distributed in Central and South America; L. williamsii, L. diffusa, and several other varieties are included in this genus. ${ }^{15-18)}$ The conven- tion on international trade in endangered species of wild fauna and flora (CITES) checklist lists only two species (L. williamsii and $L$. diffusa). ${ }^{19)}$ Classifications of Genus Lophophora are unresolved because there are only minor morphological differences between them. Even $L$. williamsii and $L$. diffusa are classified as $L$. williamsii. ${ }^{20)}$ In this manuscript, we have adopted the theory that these two species are different. L. williamsii is known by its common name, peyote. Because it contains mescaline, a psychedelic, its use is regulated in some countries. There should be the method to indentify this species but there is not because it is very difficult to differentiate those two species morphologically. The species-specific detection methods are all the more nonexistent. Thus, we have analyzed the nucleotide alignment of the chloroplast trn $\mathrm{L}$ intron region in L. williamsii and L. diffusa and clarified the differences. Three sequence patterns were observed in L. williamsii (DNA Data Bank of Japan (DDBJ) accession Nos. AB362488, AB362489, and AB362490), and one pattern was observed in L. diffusa (AB362491). We found that these two species have different sequences in the $\operatorname{trn} \mathrm{L}$ intron region. In the course of our investigation, we realized that PCR amplification using total DNA extracted from Lophophora plant as template was sometimes unsuccessful because of the polysaccharide content of the plants. We wanted to devise an amplification technique that is unaffected by impurities in the template. With this motivation, we adopted LAMP for the identification of Lophophora plants. This is the first report for the rapid detection of a psychedelic-containing plant by LAMP.

\section{MATERIALS AND METHODS}

Plant Materials Eleven specimens of Lophophora williamsii J. M. COULTER and four specimens of Lophophora diffusa Bravo from the Medicinal Plant Garden, Tokyo Metropolitan Institute of Public Health were used (Table 1). We identified the materials according to Anderson's morphological classification. ${ }^{15)}$

Designing LAMP Primers We designed two sets of 
Table 1. Plant Materials Used in This Study and Initial Rise Time of LAMP

\begin{tabular}{|c|c|c|c|c|c|c|}
\hline \multirow[b]{2}{*}{ Species } & \multirow{2}{*}{$\begin{array}{c}\text { Voucher } \\
\text { No. }\end{array}$} & \multicolumn{2}{|c|}{ Initial rise time of LAMP $(\min )^{b)}$} & \multirow{2}{*}{$\begin{array}{c}\text { Total DNA } \\
\text { concentration } \\
(\mathrm{ng} / \mu \mathrm{l})\end{array}$} & \multirow{2}{*}{$\begin{array}{c}\text { Mescaline } \\
\text { contents } \\
(\mathrm{mg} / \mathrm{g})^{c)}\end{array}$} & \multirow{2}{*}{$\begin{array}{c}\text { DDBJ accession } \\
\text { No. of } t r n \mathrm{~L} \\
\text { intron region }\end{array}$} \\
\hline & & $\begin{array}{l}\text { Primer set for } \\
\text { L. williamsii }\end{array}$ & $\begin{array}{l}\text { Primer set for } \\
\text { L. diffusa }\end{array}$ & & & \\
\hline \multirow[t]{8}{*}{ Lophophora williamsii J. M. COULTER } & Lo-2 & $47.7-63.2$ & - & 7.8 & 48.3 & AB362488 \\
\hline & Lo-3 & $49.8-59.3$ & - & 9.3 & 22.2 & AB362488 \\
\hline & Lo-6 & $49.1-58.3$ & - & 16.9 & 26.6 & AB362488 \\
\hline & Lo-7 & $53.7-59.2$ & - & 8.0 & 38.2 & AB362488 \\
\hline & Lo-8 & $50.5-54.9$ & - & 6.5 & 24.6 & AB362488 \\
\hline & Lo-9 & $53.7-56.3$ & - & 9.3 & 29.4 & AB362488 \\
\hline & Lo-10 & $49.7-61.2$ & - & 7.0 & 30.7 & AB362488 \\
\hline & Lo-11 & $50.5-58.9$ & - & 11.1 & 35.4 & AB362489 \\
\hline$(\text { L. williamsii var. decipiens CROIZ. })^{a)}$ & Lo-14 & $70.6-87.1$ & - & 7.3 & N.D. ${ }^{d)}$ & AB362490 \\
\hline 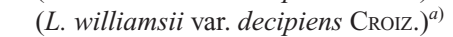 & Lo-15 & $77.6-84.3$ & - & 7.1 & N.D. ${ }^{d)}$ & AB362490 \\
\hline$(\text { L. williamsii var. decipiens CROIZ. })^{a}$ & Lo-16 & $76.5-87.3$ & - & 7.1 & N.D. ${ }^{d)}$ & AB362490 \\
\hline \multirow[t]{4}{*}{ Lophophora diffusa BRAVO } & Lo-17 & - & $75.8-82.4$ & 10.8 & N.D. ${ }^{d)}$ & AB362491 \\
\hline & Lo-18 & - & $68.9-77.6$ & 11.9 & N.D. ${ }^{d)}$ & AB362491 \\
\hline & Lo-19 & - & $79.6-82.4$ & 11.8 & N.D. ${ }^{d)}$ & AB362491 \\
\hline & Lo-20 & - & $74.6-81.1$ & 15.1 & N.D. ${ }^{d)}$ & AB362491 \\
\hline
\end{tabular}

a) These samples were identified as $L$. williamsii in this study but were identified as $L$. williamsii var. decipiens in the literature. ${ }^{16,18)}$ b) Results are given as the ranges of three examinations. c) Mescaline content was determined by HPLC, and details were described in another report (submitted). $d$ ) Not detected.

species-specific primers for L. williamsii and L. diffusa (Fig. 1). LPw-F3, LPw-B3, LPw-FIP, LPw-BIP, LP-LF and LPwLB were for L. williamsii, and LPd-F3, LPd-B3, LPd-FIP, LPd-BIP, LP-LF and LPd-LB were for L. diffusa. The primer sequences are shown in Table 2.

Real-Time Monitoring of Turbidity and Detection in LAMP of Lophophora Plants Total DNA was extracted from $20-50 \mathrm{mg}$ of plant material using a DNeasy Plant Mini Kit (QIAGEN) according to the manufacturer's instructions. DNA concentrations were measured with a DU640 spectrophotometer (Beckman). LAMP was carried out with a Loopamp DNA amplification kit (Eiken Chemical Co., Ltd.) according to the appendix with modification. Briefly, $25 \mu \mathrm{l}$ of a reaction mixture containing $1.0 \mu \mathrm{l}$ of genomic DNA, $12.5 \mu \mathrm{l}$ of $2 \times$ Reaction Mix, 8 units of Bst DNA polymerase, $0.5 \mu \mathrm{l}$ of Fluorescent Detection Reagent, $10 \mathrm{pmol}$ each of LPw-F3 (LPd-F3) and LPw-B3 (LPd-B3), 80 pmol each of LPw-FIP (LPd-FIP) and LPw-BIP (LPd-BIP), and 40 pmol each of LP-LF and LPw-LB (LPd-LB), was incubated at $60^{\circ} \mathrm{C}\left(58^{\circ} \mathrm{C}\right.$, for a set of primers for $L$. diffusa) for 90 or $120 \mathrm{~min}$ and then heated at $80^{\circ} \mathrm{C}$ for $5 \mathrm{~min}$. Real-time turbidity was monitored with Realoop-30 (Moritex Co., Ltd., Tokyo). Initial rise times were calculated by Realoop-30 from the amplification curves. After the reaction, the tubes were irradiated under a UV lamp $(254 \mathrm{~nm})$ to observe fluorescence, and $5 \mu \mathrm{l}$ of reaction mixture was electrophoresed in a $2 \%$ agarose gel containing GelRed (Biotium). Each experiment was conducted independently in triplicate runs. To confirm the sequences of the amplified products, the reaction solution was digested with MseI (NEB) and was electrophoresed.

\section{RESULT AND DISCUSSION}

Designing LAMP Primers First, we designed six primers for LAMP, including two loop primers based on the sequence from DDBJ accession No. AB362488, for the purpose of amplifying only L. williamsii DNA (Fig. 1, Table 2).
Two outer primers (LPw-F3 and LPw-B3), two inner primers (LPw-FIP and LPw-BIP), and two loop primers (LP-LF and LPW-LB) were employed in the reaction, and amplification occurred only when all primers were annealed to the target DNA. There are three nucleotide patterns in L. williamsii, and differences were observed. Compared to the topmost sequence (AB362488), four base pairs deletion were observed in Lo-11, and one base pair substitution in region $\mathrm{w}-\mathrm{F} 1 \mathrm{c}$ and ten base pairs insertion were observed in Lo-14, Lo-15, and Lo-16. The other seven regions (w-F3, F2, LFc, w-B1c, w$\mathrm{LBc}, \mathrm{B} 2$, and $\mathrm{w}-\mathrm{B} 3$ ) were shared among the three groups. One of the inner primers, LPw-FIP, was designed based on regions $\mathrm{w}-\mathrm{F} 1 \mathrm{c}$ and $\mathrm{F} 2$, so this primer did not completely anneal with Lo-14 through Lo-16. However, LPw-FIP was well matched with Lo-11 (Fig. 2). On the other hand, differences were noted in five regions between the topmost sequence (AB362488) and the sequence of L. diffusa (AB362491); there were two base pairs insertion in w-F3, two base pair deletions and one base pair substitution in w-F1c, eight base pairs insertion from nucleotide position 191 to 198 , one base pair substitution in $\mathrm{w}-\mathrm{B} 1 \mathrm{c}$, thirteen base pairs insertion in $\mathrm{w}-$ LBc, three base pair substitutions in w-B3 and one base pair substitution on nucleotide position 335. We speculated that the set of primers designed for $L$. williamsii would not anneal to the sequence of $L$. diffusa. As a result, we could design an optimal primer set for L. williamsii. Moreover, we also designed a set of primers for L. diffusa to check the selectivity of the set of primers for L. williamsii. The set of primers for L. diffusa consists of LPd-F3, LPd-B3, LPd-FIP, LPd-BIP, LP-LF and LPd-LB (Table 2). The principles of LAMP are explained in detail by Notomi et al. and Nagamine et al. ${ }^{11,21)}$

Real-Time Monitoring of Turbidity in LAMP of Lophophora Plants Turbidity derived from magnesium pyrophosphate was monitored in real time with Realoop-30. Amplifications using a set of primers for $L$. williamsii and total DNA extracted from eleven samples of $L$. williamsii were detected between 47.7 and $87.3 \mathrm{~min}$ (Fig. 3A, Table 1). In contrast, no amplification was observed when the template 


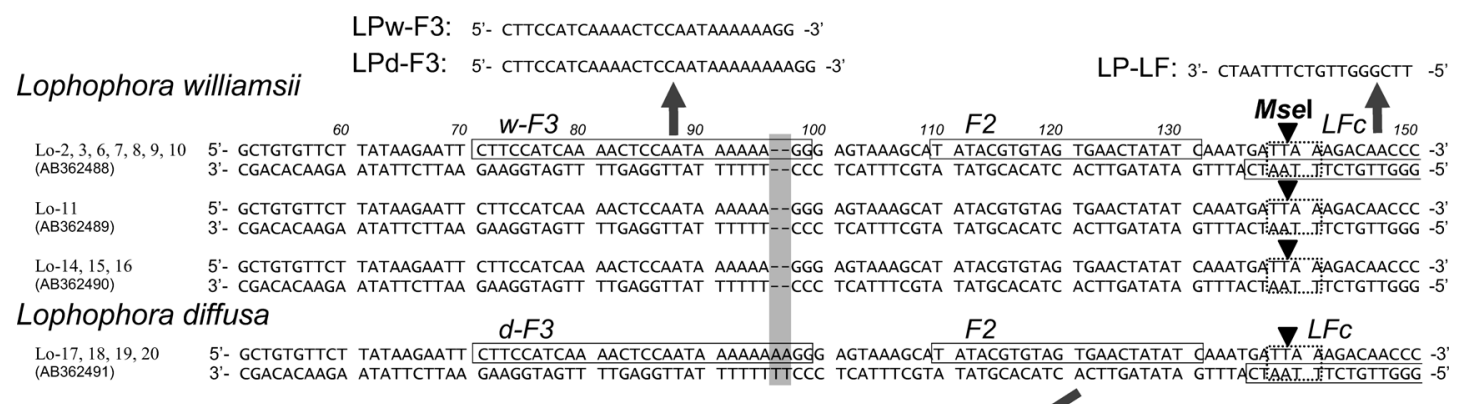

LPw-F3: 5'- cttccatcaaAactccaataaAaAagg - 3
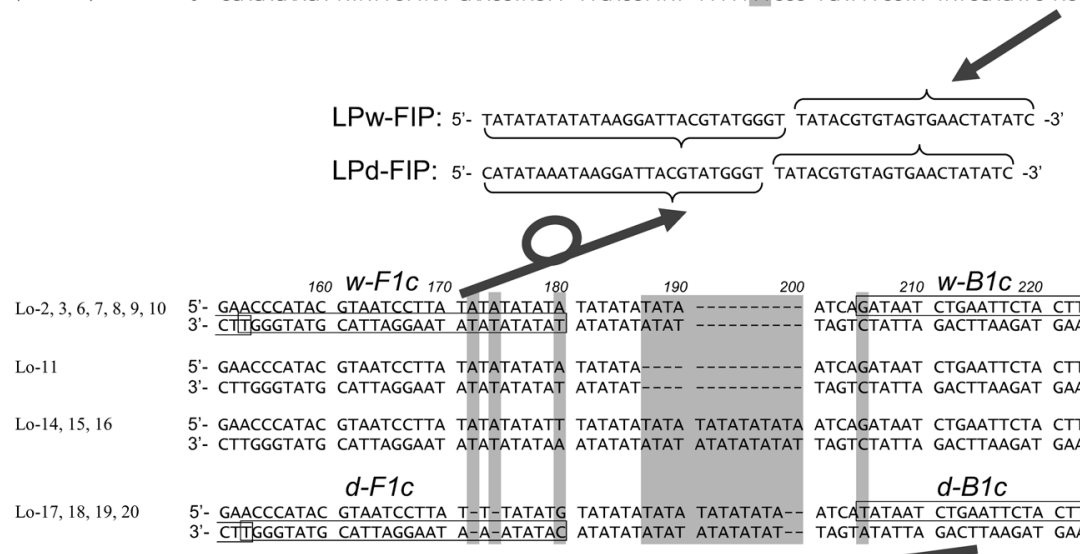

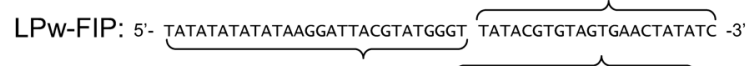

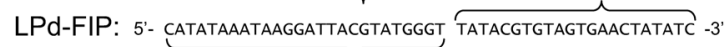

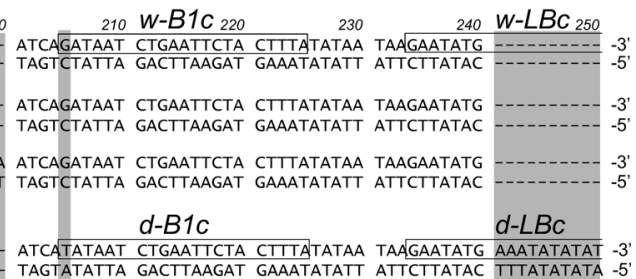

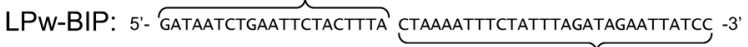

LPW-LB: 5'- gaAtatgaaAtatatattctaaAatgG - $3^{3}$

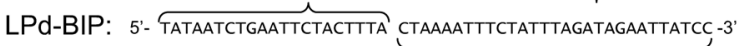

LPd-LB: 5'- gaAtaTgaAataTATATTCTAAatata -3

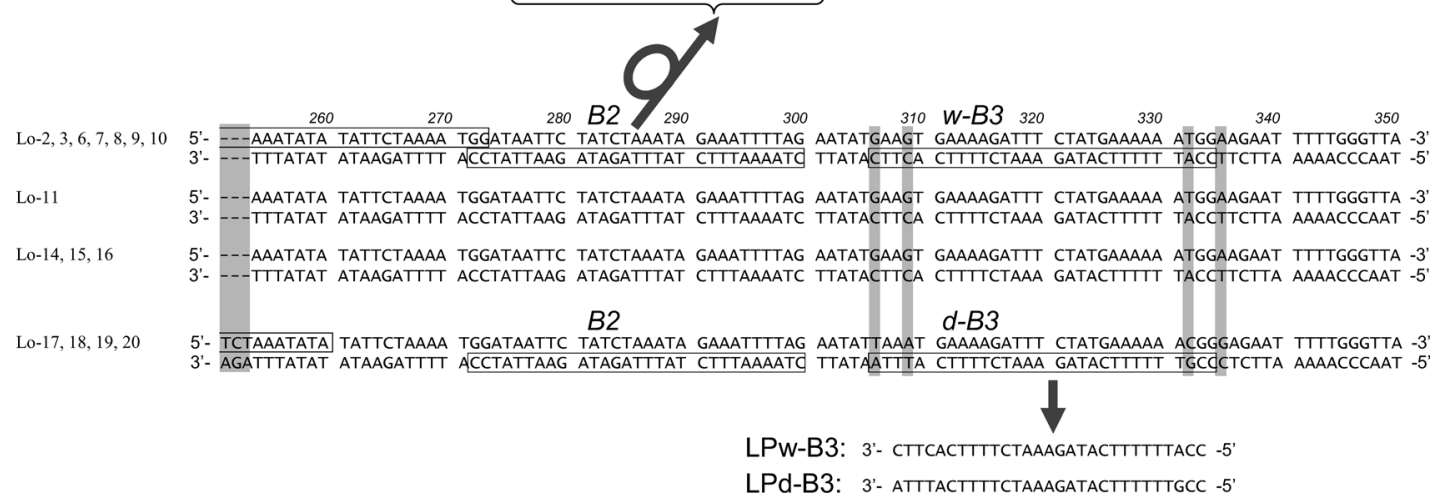

Fig. 1. Nucleotide Differences between L. williamsii and L. diffusa in Part of the trnL Intron Region and Locations of Primers Designed for LAMP

Double-stranded DNA sequences from nucleotide position 51 to 350 of the $t r n \mathrm{~L}$ intron region are shown. Dotted boxes indicated recognition site of Msel restriction enzyme. Numbers in italics above the sequences indicate aligned nucleotide positions. The shaded regions indicate nucleotide differences. Hyphens (-) denote alignment gaps. Boxed sequences indicate the primer sequences regions for primers.

Table 2. Primers Used in This Study

\begin{tabular}{|c|c|c|c|c|c|c|c|c|c|c|c|c|c|c|c|c|c|}
\hline \multirow{2}{*}{$\frac{\text { Name }^{a)}}{\text { LPW-F3 }}$} & \multicolumn{17}{|c|}{ Sequence $\left(5^{\prime}-3^{\prime}\right)$} \\
\hline & CTT & $\mathrm{CCA}$ & TCA & AAA & CTC & CAA & TAA & AAA & AGG & & & & & & & & \\
\hline LPd-F3 & CTT & CCA & TCA & AAA & CTC & CAA & TAA & AAA & AAA & GG & & & & & & & \\
\hline LPw-B3 & CCA & TTT & TTT & CAT & AGA & AAT & CTT & TTC & ACT & $\mathrm{TC}$ & & & & & & & \\
\hline LPd-B3 & CCG & TTT & TTT & CAT & AGA & AAT & CTT & TTC & ATT & TA & & & & & & & \\
\hline LPw-FIP & TAT & ATA & TAT & ATA & AGG & ATT & ACG & TAT & GGG & TTA & TAC & GTG & TAG & TGA & ACT & ATA & $\mathrm{TC}$ \\
\hline LPd-FIP & CAT & ATA & AAT & AAG & GAT & TAC & GTA & TGG & GTT & ATA & CGT & GTA & GTG & AAC & TAT & ATC & \\
\hline LPw-BIP & GAT & AAT & CTG & AAT & TCT & ACT & TTA & CTA & AAA & TTT & CTA & TTT & AGA & TAG & AAT & TAT & $\mathrm{CC}$ \\
\hline LPd-BIP & TAT & AAT & CTG & AAT & TCT & $\mathrm{ACT}$ & TTA & CTA & AAA & TTT & CTA & TTT & AGA & TAG & AAT & TAT & $\mathrm{CC}$ \\
\hline LP-LF & TTC & GGG & TTG & TCT & TTA & ATC & & & & & & & & & & & \\
\hline LPw-LB & GAA & TAT & GAA & ATA & TAT & ATT & CTA & AAA & TGG & & & & & & & & \\
\hline LPd-LB & GAA & TAT & GAA & ATA & TAT & ATT & CTA & AAT & ATA & & & & & & & & \\
\hline
\end{tabular}

a) Names beginning with the letters "LPw" are primers that anneal to the sequence of $L$. williamsii; "LPd" indicates annealing to $L$. diffusa, and "LP" indicates annealing to both species. 


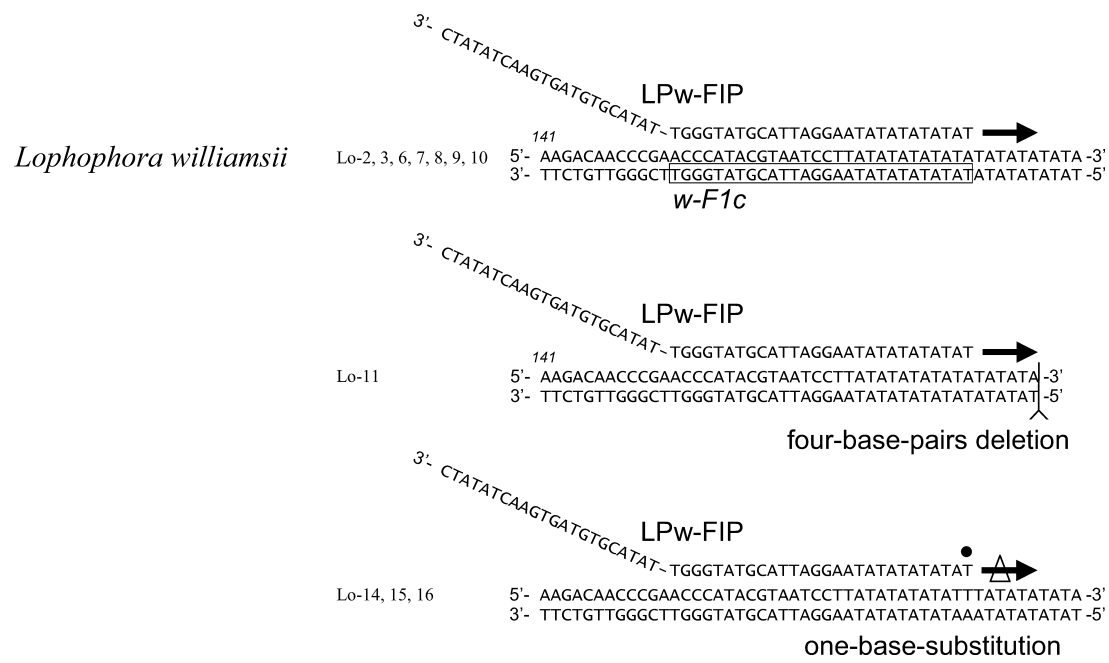

Fig. 2. Comparison of Nucleotide Alignment between LPw-FIP and Three Types of L. williamsii in the w-F1 Region Double-stranded DNA sequences from the nucleotide position 141 of $\operatorname{trn} \mathrm{L}$ intron region are shown.

A

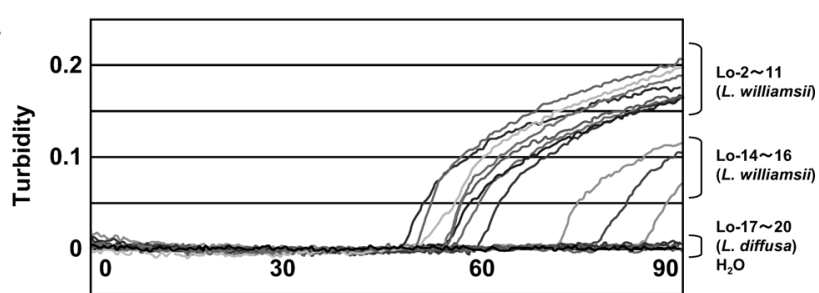

B

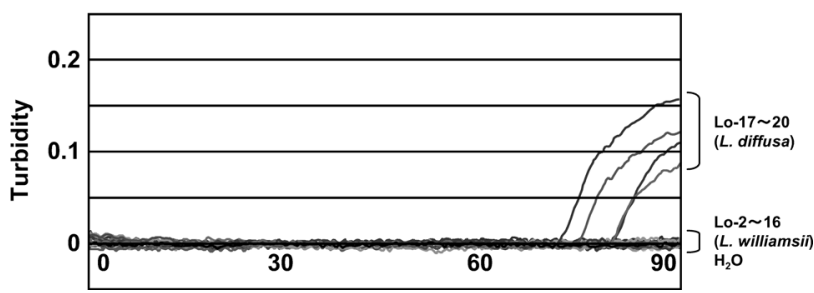

Fig. 3. Real-time Monitoring of LAMP by Turbidity Measurement in Lophophora Plants

LAMP using a set of primers for $L$. williamsii (A) and $L$. diffusa (B).

was total DNA extracted from four samples of $L$. diffusa. Although the initial rise time of Lo-11 was not delayed, those of Lo-14, Lo-15, and Lo-16 were delayed compared to those of other L. williamsii samples because LPw-FIP did not completely fit the sequence of these three samples. Interestingly, although Lo-14, Lo-15, and Lo-16 were identified as $L$. williamsii in this study, these three samples were also identified as L. williamsii var. decipiens in previous literature. ${ }^{16,18)}$ Sequence alignments in the tr $\mathrm{L}$ intron region of those three samples were different from those of Lo-2 to Lo-11. Moreover, another study of ours revealed that Lo-14, Lo-15, and Lo-16 contained no mescaline (Table 1). Using this method, we can distinguish mescaline-containing Lophophora plants from mescaline-free ones if the reaction is stopped at $65 \mathrm{~min}$. On the other hand, Lo-17 to Lo-20, which were not amplified by this set of $L$. williamsii-specific primers, were detected by a set of primers for $L$. diffusa (Fig. 3B).

Visual Detection of LAMP in Lophophora Plants LAMP could be detected visually by observing fluorescence emitted by the reaction solution under UV irradiation because fluorescent detection reagent was added to the reaction tube preliminarily. Even if no fluorescent detection reagent was added to the reaction tube, turbidity could be detected visually. Reaction tubes containing $L$. williamsii emitted fluorescence, while those containing $L$. diffusa and no template did not do so (Fig. 4, $\mathrm{A}_{1}$ ). Lo-14, Lo-15, and Lo-16, the regions with delayed amplifications (Fig. 3), were not different from Lo-2 to Lo-11. Agarose gel electrophoresis also gave the same results as fluorescence; that is, the electrophoretograms of Lo-14, Lo-15, and Lo-16 were not different from those of Lo-2 to Lo-11 (Fig. 4, $\mathrm{A}_{2}$ ). Moreover, we could confirm that the amplifications were specific because amplified products were converged a few small fragments by MseI digestions (Fig. 4, $\mathrm{A}_{3}$ ). These findings demonstrate that the results of fluorescence detection and agar gel electrophoresis were in agreement, and immediate detection of $L$. williamsii could be accomplished without special instrumentation. LAMP using a set of primers for $L$. diffusa gave the expected result (Fig. 4, $\mathrm{B}_{1-3}$ ).

Detection Limit of LAMP To investigate the detection limit and the initial rise time with changes in total DNA concentration, reactions were conducted using serially diluted DNA solutions of Lo-6. LAMP was conducted using DNA concentrations ranging from 0.05 to $10.0 \mathrm{ng}$ per tube (Fig. 5). The turbidity of reaction tubes containing $\geq 0.5 \mathrm{ng}$ DNA was well detected from $70 \mathrm{~min}$ onward, and the sample with the higher template concentration tended to show faster amplification in the same run, although the initial rise times differed slightly depending on the reaction. In samples containing $\leq 0.1 \mathrm{ng}$ of template, no amplification was observed in most occasions. If total DNA concentration of Lo-14, Lo-15 or Lo-16 was low, detection was not necessarily achieved within $90 \mathrm{~min}$. However, this did not pose any problems because we had a sufficient amount of DNA extracted by conventional techniques.

\section{CONCLUSION}

We showed that LAMP is applicable to Lophophora plants having high polysaccharide content. Compared to ARMS, PCR-RFLP, or TaqMan assay, LAMP does not require strict reaction conditions or complicated technical operation. 


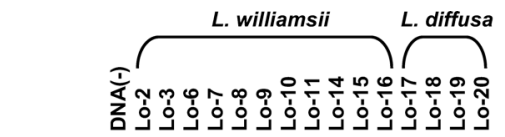

$A_{1}$

$M$
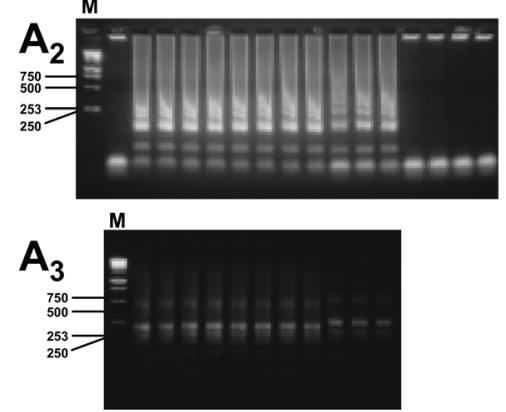

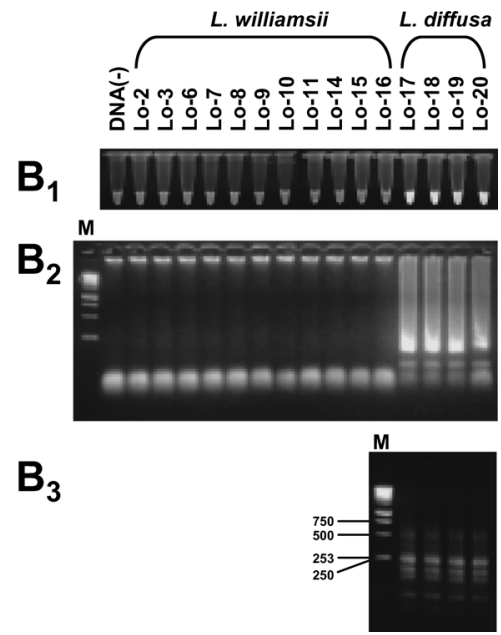

Fig. 4. Visual Inspection $\left(\mathrm{A}_{1}, \mathrm{~B}_{1}\right)$ and Agarose Gel Electrophoresis Profiles $\left(\mathrm{A}_{2}, \mathrm{~B}_{2}\right)$ of LAMP Products, and Electrophoresis Profiles $\left(\mathrm{A}_{3}\right.$, $\left.\mathrm{B}_{3}\right)$ after MseI Digestions

Images using primers specific for L. williamsii (A) and for L. diffusa (B); visual inspection was conducted under a UV lamp (254 nm). Lane M indicates $1 \mathrm{~kb}$ DNA ladder (Promega).

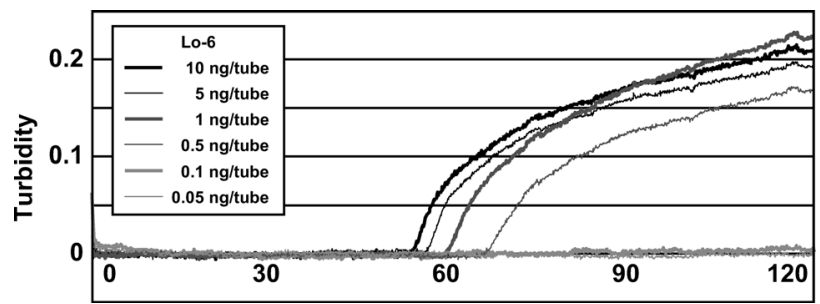

Fig. 5. Real-Time Monitoring of LAMP Using Turbidity Measurements at Different DNA Concentrations

LAMP using a set of L. williamsii-specific primers and genomic DNA of Lo-6 as template. Concentrations of template DNA were $10.0 \mathrm{ng}, 5.0 \mathrm{ng}, 1.0 \mathrm{ng}, 0.5 \mathrm{ng}, 0.1 \mathrm{ng}$, and $0.05 \mathrm{ng}$ per tube.

Moreover, no special equipment, such as a turbidity detector, is required because we have determined the optimal primer sequences and reaction conditions. This method requires only a water bath for incubation and a UV lamp for detection.

As $L$. williamsii contains mescaline, its use is regulated in some countries. Although the presence or absence of mescaline can easily be checked by chromatography, it is difficult to identify the species because not all $L$. williamsii contain mescaline. Chemotaxonomic identification of $L$. williamsii seems insufficient. DNA sequences of chloroplast $\operatorname{trn} \mathrm{L}$ intron region in Lophophora plants were revealed to be beneficial for identification and showed a good correlation with mescaline content. Based on those sequences, rapid and sensitive detection of $L$. williamsii by LAMP was achieved.

Acknowledgements We are grateful to Dr. Ryohei Yanoshita, Institute of Medicinal Chemistry, Hoshi University, for permitting us to use the DU640 spectrophotometer. This work was supported by a Grant-in-Aid from the Otani Memorial Research Foundation.

\section{REFERENCES}

1) Jun W., Elizabeth A. Z., Mol. Phylogenet. Evol., 6, 167-177 (1996).

2) Cao H., Sasaki Y., Fushimi H., Komatsu K., Biol. Pharm. Bull., 24, 1389-1394 (2001).

3) Barkmann T. J., Lim S. H., Salleh K. M., Nais J., Proc. Natl. Acad. Sci. U.S.A., 101, 787-792 (2004).

4) Gong W., Fu C. X., Luo Y. P., Qiu Y. X., Planta Med., 72, 650-652 (2006).

5) Zhu S., Fushimi H., Han G., Tsuchida T., Uno T., Takano A., Komatsu K., Biol. Pharm. Bull., 30, 527-531 (2007).

6) Newton C. R., Graham A., Heptinstall L. E., Powell S. J., Summers C., Kalsheker N., Smith J. C., Markham A. F., Nucleic Acids Res., 17, 2503-2516 (1989).

7) Sorscher E. J., Huang Z., Lancet, 337, 1115-1118 (1991).

8) Livak K. J., Genet. Anal., 14, 143-149 (1999).

9) Mizukami H., Okabe Y., Biol. Pharm. Bull., 22, 765-766 (1999).

10) Sasaki Y., Cao H., Fushimi H., Komatsu K., Biol. Pharm. Bull., 25, 1593-1599 (2002).

11) Notomi T., Okayama H., Masubuchi H., Yonekawa T., Watanabe K., Amino N., Hase T., Nucleic Acids Res., 28, e63 (2000).

12) Kuboki N., Inoue N., Sakurai T., Cello F. D., Grab D. J., Suzuki H., Sugimoto C., Igarashi I., J. Clin. Microbiol., 41, 5517-5524 (2003).

13) Annaka T., Kojima T., Ikedo M., Furuhata K., J. Antibact. Antifung. Agents, 32, 195-201 (2004).

14) Sasaki Y., Nagumo S., Biol. Pharm. Bull., 30, 2229-2230 (2007).

15) "Peyote, The Divine Cactus," ed. by Anderson E. F., The University of Arizona Press, Arizona, 1980, pp. 179-189.

16) "The Great Lexicon of Cactaceae, 266 Genera and Their Species," ed. by Ito Y., Mirai-sha, Tokyo, 1988, pp. 577-578.

17) "Cactus Handbook," ed. by Sato T., Japan Cactus Planning Co. Press, Fukushima, 1996, pp. $144-145$.

18) "Cactus and Succulent Plant," ed. by Hirao H., Kodama E., NHK Press, Tokyo, 1999, pp. 93-94.

19) “CITES Cactaceae Checklist," 2nd ed., ed. by David H., Royal Botanic Gardens Kew, London, 1999, p. 74.

20) "The Plant-Book: A Portable Dictionary of the Vascular Plants," 2nd ed., ed. by Mabberley D. J., Cambridge University Press, Cambridge, 1997, p. 420.

21) Nagamine K., Hase T., Notomi T., Mol. Cell. Probes, 16, 223-229 (2002). 